\title{
Editorial: Securing Internet of Things Through Big Data Analytics
}

\author{
Muhammad Alam ${ }^{1} \cdot$ Ting Wu$^{2} \cdot$ Fazl Ullah $^{3} \cdot$ Yuanfang Chen ${ }^{2}$ \\ Published online: 24 May 2019 \\ (C) Springer Science+Business Media, LLC, part of Springer Nature 2019
}

The "IoT" heralds the connections of a nearly countless number of devices to the Internet thus promising accessibility, boundless scalability, amplified productivity and a surplus of additional paybacks [1]. The hype surrounding the IoT and its applications is already forcing companies to quickly upgrade their current processes, tools, and technology to accommodate massive data volumes and take advantage of insights. Since there is a vast amount of data generated by the IoT, a well-analyzed data is extremely valuable. However, the large-scale deployment of IoT will bring new challenges and IoT security is of them. The philosophy behind machine learning is to automate the creation of analytical models in order to enable algorithms to learn continuously with the help of available data. Continuously evolving models produce increasingly positive results, reducing the need for human interaction. These evolved models can be used to automatically produce reliable and repeatable decisions. Today's machine learning algorithms comb through data sets that no human could feasibly get through in a year or even a lifetime's worth of work. As the IoT continues to grow, more algorithms will be needed to keep up with the rising sums of data that accompany this growth. One of the main challenge of the IoT security is the integration with communication, computing, control, and physical environment parameters to analyze, detect and defend cyber-attacks in the distributed IoT systems.

European Alliance for Innovation (EAI) took a step towards the realization of Future Intelligent Vehicular Technologies based on dependable and real-time communication, IoT, Big data and other related technologies and invite both academic and industrial research community by organizing the 2 nd edition of Future $5 \mathrm{~V}$ conference

\footnotetext{
Muhammad Alam

alam@av.it.pt

1 Instituto de Telecomunicações, Campus Universitário de Santiago, 3810-193 Aveiro, Portugal

2 School of Cyberspace, Hangzhou Dianzi University, Hangzhou, China

3 Abdul Wali Khan University Mardan, Mardan, Pakistan
}

in Islamabad, Pakistan. Future $5 \mathrm{~V}$ is an annual international conference by EAI (European Alliance for Innovation) and co-sponsored by Springer. Future $5 \mathrm{~V}$ attracted more than 150 research articles in field of Vehicular networks/communications covering theory and practices in the after mentioned field of study. This special issue was organized for the extended and invited papers from Future5V conference held in Islamabad, Pakistan. Following are the details of accepted papers in this special issue.

The first paper tittled "Image Steganalysis via Random Subspace Fisher Linear Discriminant Vector Functional Link Network and Feature Mapping" presents a comprehensive steganography (the hiding of data within a cover) [2]. A new algorithm for processing feature that makes two optimizations into a random vector functional link (RVFL) network is proposed in this paper. The first optimization locates the processing phase of RVFL, where we model the eigenspectrum by the eigenvalue distribution of the scatter matrix. This eigenspectrum is used to generate the transpose matrix and obtain final features after feature reduction. The second optimization is the use of the random subspace Fisher linear discriminant (FLD) instead of random weights in RVFL. The weights between the input and enhancement nodes more accurately represent the relative importance of the features. In the experiments, the authors have compared the performance of other classifiers with the proposed method using five high-dimensional features. It is shown that the proposed method outperforms other classifiers in these steganalysis methods.

The 2nd accepted paper is about evolutionary game theoretic incentive mechanism to promote the cooperation of individual users to curb the expansion of unwanted traffic [3]. The existence of malicious users seriously undermine user privacy and network security by distributing a large amount of unwanted traffic, such as spam, popup, and malware. This, to some extent, can be identified with the cooperation of individual users by installing anti-virus toolkits. However, users need to purchase such software at an additional cost. Therefore, unless builtin incentive mechanisms exist, rational users will choose not to install virus software. If enough network entities 
behave in this way, the network will be flooded with unwanted traffic. Therefore, in this paper, the authors proposed a combined reward and punishment mechanism to further incentivize cooperative behavior. Meanwhile, the acceptance condition of the proposed framework is analyzed and a number of simulations are carried out to evaluate the acceptance conditions of our framework. The experimental results indicate that reward and punishment mechanism can efficiently incentivize users to adopt cooperative behavior and reduce unwanted traffic.

The third accepted paper presents a comprehensive work on data aggregation technique that eliminates redundancy in the sensed data [4]. In Wireless Sensor Networks (WSNs), a dense deployment of sensor nodes produce data that contain intra-temporal and inter-spatial correlation. To reduce the intensity of correlation, the authors proposed innode data aggregation technique that eliminates redundancy in the sensed data in an energy-efficient manner. A novel data-driven approach is adopted to perform in-node data aggregation using an underlying cluster-based hierarchical network. The proposed approach partially processes the data at each member node and forwards a fraction of the actual data, i.e., fused data, towards the cluster head. At each member node, the raw captured data is categorized into various classes, i.e., stratum. Each member node continuously senses the environment for temperature readings and compares them with the mean values of various strata. If the temperature reading is less than or greater than the mean value, it is compared with the existing Min/Max of that particular stratum. If in case, the new reading is less than/greater than the Min/Max of a particular stratum, it replaces these values, accordingly. The proposed approach is computationally lightweight, energy-efficient and reduces the degree of correlation among the resourceconstrained sensor nodes. As as a result, communication cost, packet collision and network congestion are reduced and the network lifetime is enhanced.

The next accepted paper is about the energy efficient routing and channel borrowing techniques [5]. In Wireless Sensor Networks (WSNs), energy-efficient routing is required to conserve the scarce resources of these networks [6]. Various energy-efficient routing protocols have been proposed in this context. Among them, cluster-based hierarchical routing protocols are the most efficient ones. In these protocols, each sensor node, i.e., a member node, continuously captures the data from environment, stores in its buffer and waits for its allocated timeslot to transmit the data to a designated cluster head. The buffer of each node is subject to one or more pre-defined threshold values. If the buffer occupancy level exceeds the threshold value, the node starts to drop the captured data. A member node may continuously capture the data and may have to wait longer than expected to transmit it to the base station. In this context, the data generated by the underlying application may suffer excessive delay and may no longer be useful for further analysis. To address this issue, we propose a channel borrowing scheme for the member nodes within each cluster. Each member node monitors its buffer and if the occupancy level exceeds a threshold value, a channel borrowing request is initiated. Each node maintains a table of potential cluster heads. A neighboring cluster head is selected for channel borrowing, based on its signal strength. If a neighboring cluster head has a spare channel, it is allocated to the member node. The request for borrowing a channel must be acknowledged within a predefined threshold waiting time. The experimental results show that our propose scheme reduces packet loss, energy consumption, end-to-end delay and enhances throughput and network lifetime.

In the next accepted paper, an image secret sharing (ISS) scheme with no third-party scrambling method is proposed and evaluated [7]. Values such as cellular sum in initial configurations and neighbor radius can potentially affect confusion and diffusion in cellular automata. Specifically, both confusion and diffusion characteristics in cellular automata usually are not sufficient to break up the high correlation between the secret image pixels when these two parameters are too small. Thus, using only One-Dimensional Reversible Memory Cellular Automata (ODRM-CA) to generate shadow images will lead to the leakage of original secret image's information by some shadow images. In order to solve this problem, in this paper, we propose an image secret sharing (ISS) scheme with no third-party scrambling method. This is realized by increasing the values of cellular sum and neighbor radius to implement the scrambling process for the secret image, thus both processes of image scrambling and image secret sharing can be achieved by ODRM-CA. Experimental results show that this scheme can generate strong random shadow images to avoid leaking information of the original secret image.

In the last accepted paper, a novel external corrosion risk online perception method is proposed to solve the dangerous external corrosion threat and supply a measurable safe risk perception ability for the industrial safe Internet of Things (IoTs) with the infrared thermal wave as the direct sensors [8]. The three layers model is established with direct variables measuring layer, external corrosion risk soft measuring layer and monitoring cycle decisionmaking layer. And in the direct variable measuring layer the infrared thermal wave is applied to measure the three direct variables, area ratio of cladding defects,cladding layer thickness reading and overlapping between external and internal corrosion defects, in the direct variables measuring layer. In the external corrosion risk soft measuring layer and monitoring cycle decision-making layer, external corrosion 
risk can be soft measured through a cladding-conditionbased risk matrix and the most optimal monitoring cycle can also be determined through a decision-making tree based on the three direct variables.

\section{References}

1. Bartolomeu P, Alam M, Ferreira J, Fonseca JA (2018) Supporting deterministic wireless communications in industrial IoT. IEEE Trans Ind Inf 14(9):4045-4054. https://doi.org/10.1109/tii.2018. 2825998

2. Fan L, Sun W, Feng G (2018) Image steganalysis via random subspace fisher linear discriminant vector functional link network and feature mapping. Mobile Networks and Applications. https://doi.org/10.1007/s11036-018-1167-z

3. Liu J, Li M, Alam M, Chen Y, Wu T (2019) A game theoretic reward and punishment unwanted traffic control mechanism. Mobile Networks and Applications. https://doi.org/10.1007/s110 36-018-1166-0

4. Jan SRU, Jan MA, Khan R, Ullah H, Alam M, Usman M (2018) An energy-efficient and congestion control data-driven approach for cluster-based sensor network. Mobile Networks and Applications. https://doi.org/10.1007/s11036-018-1169-x

5. Khan H, Jan MA, Alam M, Dghais W (2018) A channel borrowing approach for cluster-based hierarchical wireless sensor networks. Mobile Networks and Applications. https://doi.org/10.1007/s110 36-018-1171-3

6. Alam M, Rodriguez J (2010) A dual head clustering mechanism for energy efficient WSNs. Mobile Lightweight Wireless Syst, 380-387. https://doi.org/10.1007/978-3-642-16644-0_33

7. Hu W, Yao Y, Zhou D, Zheng Q, Choo K-KR (2018) A novel image secret sharing scheme without third-party scrambling method. Mobile Networks and Applications. https://doi.org/10.1007/s110 36-018-1168-y

8. Cong G, Lu D, Lv Y, He Y (2018) A novel industrial safety IoTs architecture for external corrosion perception based on infrared. Mobile Networks and Applications. https://doi.org/10.1007/s110 36-018-1170-4

Publisher's Note Springer Nature remains neutral with regard to jurisdictional claims in published maps and institutional affiliations.

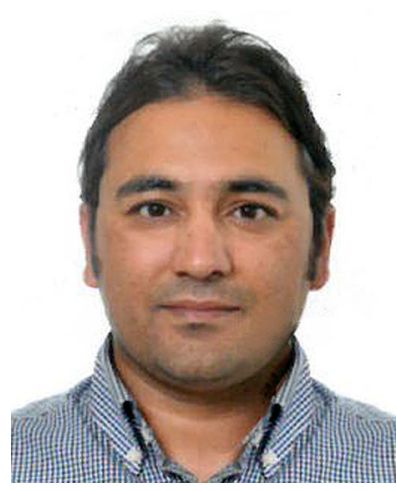

Muhammad Alam holds a Ph.D. degree in computer science from University of Aveiro, Portugal (2009 - 14). In 2009, he joined the Instituto de Telecomunica ções - Aveiro (Portugal) as researcher and completed his Ph.D. from University of Aveiro. He has participated in several European Union FP7 projects such as Hurricane, C2POWER, ICSI, PEACE and Portuguese government funded projects such SmartVision. Currently, he is working as senior researcher at Instituto de Telecomunica?c?oes and participating in European Union and Portuguese government funded projects. His research interests include Real-time wireless communication, 5G, Vehicular networks, Context-aware systems and Radio resource management in next generation wireless networks. He is the author of several journal and conference publications as well as book chapters. He is also the TPC member and reviewer for a number of reputed conferences, journals, and magazines. He is IEEE senior member. He served as general co-chair of future $5 \mathrm{~V}$ conference and also served as session chairs in a number of conferences.

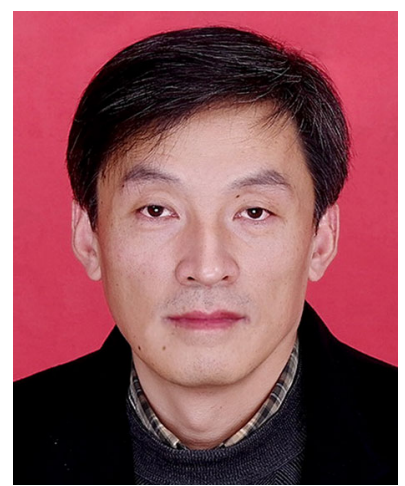

Professor Ting $\mathrm{Wu}$ received his Ph.D. degree from Shandong University, China. $\mathrm{He}$ is currently serving as full Professor and Dean of the School of Cyberspace of Hangzhou Dianzi University, Hangzhou, China. He has actively participated in several national funded projects as principal investigator and senior researcher. $\mathrm{He}$ is the author of a number of conference and journal publications and served as General co-chair of the IEEE ICCCN conference

in 2018. His research interests are Computer and information security.

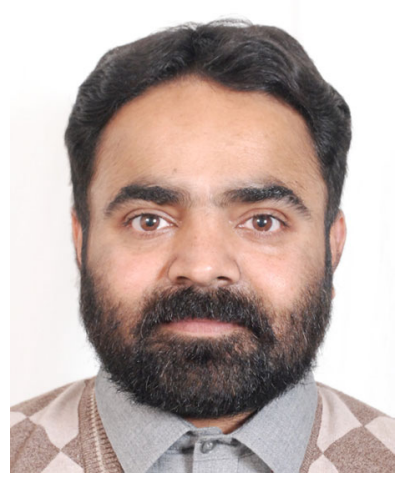

Fazl Ullah is an Assistant Professor at the Department of Computer Science, Abdul Wali Khan University Mardan, Pakistan. He is a gold medalist, and had been the recipient of various prestigious scholarships during his studies. He was the recipient of MEXT scholarship, endowment fund, and merit scholarship. His research interests are cross layer signaling and performance analysis of Mobile Ad Hoc Networks, Cognitive Radio Networks, and Internet of Things. Recently, he has been involved in latest developments in the field of Internet of Vehicles security and privacy issues, Cognitive Radio Internet of Vehicles, and Big Data Analytics. He has published his research work in various IEEE and ACM/Springer International conferences and Journals. Recently, he has been chair of various conferences and special sessions such as OS-RAS-2019, Future5V-2017, CCODE-2017, IoT-BC2-2016. He has been an active reviewer for high cited and highly ranked international journals, including Springer MONET, IET Wireless Sensor Systems, IEEE Communication Magazine, IEEE Transaction on ITS, Elsevier FGCS, Concurrency and Computation: Practice and Experience and so on. 


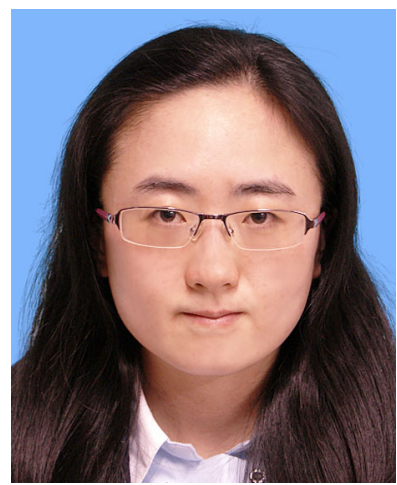

Yuanfang Chen is currently a Distinguished Professor with the Hangzhou Dianzi University, China. She received the M.S. and the first Ph.D. degrees from the Dalian University of Technology, China, and the second Ph.D. degree from the University Pierre and Marie CURIE, France. From 2009 to 2010, she was an Assistant Researcher with the Illinois Institute of Technology, USA, with Prof. Xiangyang Li. Her research interests include localization, deep/machine learning, algorithm design, and wireless sensor networks. She was a Travel Grant recipient of MASS 2009, IWCMC 2009, and SIGCOMM 2013. She has served as a volunteer of MobiCom 2010 and MobiHoc 2010, and IEA-AIE 2012. She has been invited as the Session Chair of conferences, including MobiQuitous 2013, ICA3PP 2015, and CODIT 2018, and the TPC member of Globecom 2014 and MobiApps 2014. She has served as the Fixed Reviewer of top journals and conferences, including IEEE TRANSACTIONS ON INDUSTRIAL INFORMATICS, ACM Computing Surveys, IEEE TRANSACTIONS ON FUZZY SYSTEMS, and AD HOC and; SENSOR WIRELESS NETWORKS. She is currently an Associate Editor of the EAI Endorsed Transactions on Industrial Networks and Intelligent Systems. 